\title{
FESTULOLIUM METABOLITES ACCUMULATION RESPONSE TO PHOTOPERIOD OF FLOWERING TERMOINDUCTION
}

Jolanta LELIŪNIENĖ, Institute of Agriculture and Food Science, Faculty of Agronomy, Aleksandras Stulginskis University. Studentų g. 11, Akademija LT-53361, Kauno r., Lithuania. jolanta.leliuniene@asu.lt

Ligita BALEŽENTIENĖ, Institute of Environment and Ecology, Faculty of Forest Sciences and Ecology, Aleksandras Stulginskis

University. Studentų g. 11, Akademija LT-53361, Kauno r., Lithuania. ligita.balezentiene@ asu.lt (corresponding author)

Evaldas KLIMAS, Institute of Agriculture and Food Science, Faculty of Agronomy, Aleksandras Stulginskis University. Studentu g. 11, Akademija LT-53361, Kauno r., Lithuania. evaldas.klimas@asu.lt

Most of plant development, physiological and metabolic processes are regulated by not only soluble sugars such as glucose and sucrose, but also by other signal molecules, such as phytohormones.

The investigation of flowering induction, considering the influence of vernalisation duration and photoperiod on morphogenesis stages and accumulation metabolites in the new Festulolium cultivars 'Vètra' and 'Punia' was carried out at the phytotron complex of the Plant Physiology Laboratory, Institute of Horticulture, Lithuanian Research Centre for Agriculture and Forestry in 2011-2012.

The data revealed impact of vernalisation and photoperiod on accumulation of both types of assessed metabolies, i.e. phytohormones and saccharides, and thus confirmed their substantial role. 90 short-day vernalisation induced the highest total phytohormone content in 'Vètra', when plant achieved tillering stage and was going for intensive growth when growth regulators will be important in the metabolic regulation.

The highest phytohormone content was recorded after long - day 130+20 day vernalization at VII and IV organogenesis stages of 'Vètra' and 'Punia' respectively.

Saccharides content significantly depended on photoperiod and temperature during vernalisation and was different in 'Vètra' and 'Punia'.

Keywords: Festulolium, phytohormones, saccharides.

\section{INTRODUCTION}

Growth and reproductive development of the Festulolium plant are regulated by a complex set of interacting environmental factors. Flowering induction of overwintering Festulolium required the low-temperatures or vernalisation (Michaels, Amasino, 2000). This process also might be called thermoinduction (Duchovskis, 2004). Nonetheless, conditions of flowering induction often act by means of metabolites. Saccharides are sensitive to photoperiod of prefloral induction. Sucrose is rapidly transferred to apical meristems and induced its growth and development (Samuoliene et al., 2008). Glucose and fructose greatly affect the formation and structure of the plant cells and whole body metabolism. Once the plant has response to a number of stressors saccharides are involved as a nutrient and signal transmission molecules which are activating the specific hormonal and non-hormonal signalling pathways that lead to important modifications of gene expression and enzymes activity (Koussevitzky et al., 2007). Moreover, most of plant development, physiological and metabolic processes are regulated by not only soluble sugars such as glucose and sucrose, but also by other signal molecules, such as phytohormones (Gibson, 2004; Halford et al., 2010). In some cases, response reaction of saccharides and phytohormones may affect the nature of regulation of a number of physiological processes. Sugars act quantity, location and / or transmission of phytohormones. Some scientists consider that hexose affects the metabolism and / or transmission of phytohormones (Cheng et al., 2002; Gibson, 2004). Phytohormones coordinate development, proportions and interaction of all plant structures. Though content of free phytohormones varies during different stages of flowering initiation, but their effect has synergistic or antagonistic nature (Bernier et al., 1993). Phytohormones regulate plant' response to stress, and their amount determinates plant development processes (Javid et al., 2011). It was found that phytohormones together with metabolites in certain ratios can self-activate genes of evocation and flower initiation. Morphogenetic studies of apical meristem culture in vitro have shown that well-known phytohormones induces generative development of perennial herb. However, the question how much each of them are involved in flowering initiation processes is not answered yet (Duchovskis, 2004).

For the production grass seed it is essential to ensure a stable seed yield. Therefore, the study was designed to investigate the conditions of flowering induction, considering the influence of vernalisation duration and photoperiod on morphogenesis stages and formation of prefloral elements of the new Festulolium cultivars.

Copyright (C) 2017 The Authors. Published by Aleksandras Stulginskis University. This is an open-access article distributed under the terms of the Creative Commons Attribution License (CC-BY 4.0), which permits unrestricted use, distribution, and reproduction in any medium, provided the original author and source are credited. 


\section{MATERIAL AND METHODS}

Festulolium cultivars 'Vètra' and 'Punia' are enlisted in the National list (2008) and were investigated at the Plant Physiology Laboratory of phytotron complex, the Institute of Horticulture, Lithuanian Research Centre for Agriculture and Forestry, in 2011-2012. Five plants of each cultivar were sown in each 5 L pot in peat substrate (pH 6-6.5) in three replications. The plants were grown in greenhouse till the tillering stage at the temperature of $20 \pm 2{ }^{\circ} \mathrm{C}$ and 16 hours photoperiod. Later 3 pots kept in greenhouse and others were moved to $4{ }^{\circ} \mathrm{C}$ low temperature growth chambers for 90 , 110 and 130 days for passing of vernalisation processes, where the 8 and 16 hour (h) photoperiods were maintained. After vernalisation periods plants were transferred to a greenhouse $\left(20 \pm 2{ }^{\circ} \mathrm{C}\right.$ and 16 hours photoperiod) for additional 20 days.

Growth and organogenesis stages (I-XII) were identified in lines with Kuperman et al. (1982). Biometric parameters, namely plant height, shoot number, and dry mass were measured and analysis of phytohormones and saccharides were performed after each period in greenhouse and climatic chambers.

Extraction of phytohormones was performed after modified Wang et al. (2003) method. $1 \mathrm{~g}$ of green plant mass was crushed in liquid nitrogen, extracted with $10 \mathrm{ml}$ of isopropanol for 24 hours. Extract was centrifuged for 5 min at $2500 \mathrm{rpm}$. The residue was washed 3 times with isopropanol. The sample evaporated in a rotary evaporator (BUCHI B-490, Switzerland). The collected fractions were concentrated using a solid phase extraction method and $\mathrm{NH}_{2}$-cartridges. The prepared samples were store at $+4{ }^{\circ} \mathrm{C}$. Quantitative analysis of jazmonic acid (JR), aminocyclopropane-carboxylic acid (ACKA), indole-3-butyric acid (IBA), benzyl adenine (BA), indole-3-acetic acid (IAA), abscisic acid (ABA), cinnamic acid (CA), gibberellic acid (GA4) and zeatin, was performed using liquid chromatography system (Shimadzu Prominence UFLC, Japan) with mass spectrometer (LCMS 2020), column YMC Triart C18 (250 mm $4.6 \mathrm{~mm}, 5 \mathrm{~mm}$ ) (YMC, Japan). Mobile phase was methanol and deionized water (1:1) with $0.25 \%$ acetic acid. Flow rate $-0.3 \mathrm{ml} \mathrm{min}^{-1}$.

For the effective liquid chromatography (HPLC) 1-2 g samples of fresh mass were crushed, diluted with 4 ml of doubledistilled water, then extracted for 24 hours and filtered using $0.45 \mathrm{~mm}$ cellulosic filter before HPLC analysis. Fructose, glucose, raffinose, sucrose and maltose identified by chromatograph (Shimadzu HPLC10A, Japan) with a refractive index detector (RID-10A), column thermostat (CTO-10 AS VP), de-gazer (DGU-14A) and a pump (LC-10 AT OP). Saccharides distribution performed using 300x4.6 nm column (Shodex Sugar SC1011, Japan) at $80{ }^{\circ} \mathrm{C}$. Mobile phase was double-distilled water. Saccharides identified by matching their spectral characteristics against those of standard curves.

Statistical analysis. The confidence limits of the data were based on dispersion analyse method and evaluated using the ANOVA post-hoc Fisher $F$-criterion test. Standard error between treatments evaluated using MS Excel. The results were statistically evaluated using the statistical package STATISTICA of Stat Soft.

\section{REZULTS AND DISSCUSION}

The research data revealed impact of vernalisation and photoperiod on Festulolium both types of assessed metabolies, i.e. phytohormones and saccharides accumulation, and thus confirmed their substantial role.

After 90 short-day vernalisation the highest total phytohormone content was recorded in 'Vètra', when plant achieved tillering stage and was going for intensive growth when growth regulators will be important in the metabolic regulation (Table 1).

Table 1. Impact of photoperiod and vernalisation on phytohormon accumulation $\left(\mu \mathrm{g} \mathrm{g}^{-1}\right)$ in 'Vètra' ir 'Punia' fresh mass

\begin{tabular}{|c|c|c|c|c|c|c|c|c|c|c|}
\hline \multicolumn{2}{|r|}{ Vernalisation } & \multicolumn{9}{|c|}{ 'Vètra' } \\
\hline Duration, day & Photoperiod, h; Temperature, ${ }^{\circ} \mathrm{C}$ & JA & ACKA & Zeatin & IBA & $\mathrm{BA}$ & IAA & $\mathrm{ABA}$ & $\mathrm{CA}$ & GA4 \\
\hline \multirow[t]{3}{*}{90} & $8 \mathrm{~h}-4^{\circ} \mathrm{C}$ & 0.82 & 0.13 & 0.01 & 0.47 & 0.08 & 0.21 & 0.01 & 0.01 & 0.44 \\
\hline & $16 \mathrm{~h}-4^{\circ} \mathrm{C}$ & 0.70 & 0.09 & 0.01 & 0.25 & 0.06 & 0.12 & 0.01 & 0.06 & 1.50 \\
\hline & $16 \mathrm{~h}-20^{\circ} \mathrm{C}$ (greenhouse) & 0.95 & 0.11 & 0.01 & 0.30 & 0.12 & 0.26 & 0.01 & 0.03 & 0.49 \\
\hline \multirow[t]{3}{*}{130} & $8 \mathrm{~h}-4^{\circ} \mathrm{C}$ & 0.34 & 0.02 & 0.01 & 0.10 & - & - & - & - & 0.12 \\
\hline & $16 \mathrm{~h}-4^{\circ} \mathrm{C}$ & 0.39 & 0.03 & 0.01 & 0.14 & - & - & - & - & 0.31 \\
\hline & $16 \mathrm{~h}-20^{\circ} \mathrm{C}$ (greenhouse) & 0.31 & 0.05 & 0.01 & 0.05 & - & - & - & - & 0.27 \\
\hline \multirow[t]{3}{*}{$130+20$} & $8 \mathrm{~h}-4^{\circ} \mathrm{C}$ & 0.59 & 0.11 & 0.01 & 0.52 & 0.01 & 0.02 & - & 0.05 & 1.05 \\
\hline & $16 \mathrm{~h}-4^{\circ} \mathrm{C}$ & 0.99 & 0.45 & 0.01 & 0.61 & 0.01 & 0.06 & - & 0.04 & 0.90 \\
\hline & $16 \mathrm{~h}-20^{\circ} \mathrm{C}$ (greenhouse) & 0.53 & 0.06 & 0.01 & 0.32 & 0.03 & 0.09 & - & 0.04 & 1.15 \\
\hline & Vernalisation & \multicolumn{9}{|c|}{ 'Punia' } \\
\hline Duration, day & Photoperiod, h; Temperature, ${ }^{\circ} \mathrm{C}$ & JA & ACKA & Zeatin & IBA & BA & IAA & $\mathrm{ABA}$ & $\mathrm{CA}$ & GA4 \\
\hline \multirow[t]{3}{*}{90} & $8 \mathrm{~h}-4^{\circ} \mathrm{C}$ & 1.12 & 0.16 & 0.01 & 0.23 & 0.05 & 0.13 & - & 0.01 & 0.48 \\
\hline & $16 \mathrm{~h}-4^{\circ} \mathrm{C}$ & 1.15 & 0.15 & 0.01 & 0.18 & 0.04 & 0.12 & - & 0.07 & 3.05 \\
\hline & $16 \mathrm{~h}-20^{\circ} \mathrm{C}$ (greenhouse) & 0.67 & 0.08 & 0.01 & 0.17 & 0.04 & 0.14 & - & 0.01 & 0.54 \\
\hline \multirow[t]{3}{*}{130} & $8 \mathrm{~h}-4^{\circ} \mathrm{C}$ & 0.30 & 0.06 & 0.01 & 0.14 & - & - & - & - & 0.18 \\
\hline & $16 \mathrm{~h}-4^{\circ} \mathrm{C}$ & 0.23 & 0.15 & - & 0.13 & - & - & - & - & 0.25 \\
\hline & $16 \mathrm{~h}-20^{\circ} \mathrm{C}$ (greenhouse) & 0.37 & 0.04 & 0.01 & 0.18 & - & - & - & - & - \\
\hline \multirow[t]{3}{*}{$130+20$} & $8 \mathrm{~h}-4^{\circ} \mathrm{C}$ & 0.40 & 0.04 & 0.01 & 0.16 & - & - & - & 0.01 & 0.57 \\
\hline & $16 \mathrm{~h}-4^{\circ} \mathrm{C}$ & 0.73 & 0.31 & 0.01 & 0.19 & 0.01 & 0.02 & - & 0.02 & 0.49 \\
\hline & $16 \mathrm{~h}-20^{\circ} \mathrm{C}$ (greenhouse) & 0.79 & 0.09 & 0.01 & 0.44 & - & 0.02 & 0.01 & 0.03 & 1.08 \\
\hline
\end{tabular}


Nonetheless, the highest phytohormone content was recorded after long -day 130+20 day vernalization at VII and IV organogenesis stages of 'Vètra' and 'Punia' respectively (Table 1, 4, 7).

The highest GA4 content was detected after 90 long-day period, when plants achieved II and III organogenesis stages. This hormone regulates seed germination, leaf growth, stem elongation and flowering (Magome et al., 2004). It is acknowledged, that GA and ABA interact in some controlled processes. Moreover, they can interact also with sucrose as they are involved in enzymes regulation and saccharides metabolism (Bernier et al., 1993).

Significant the highest IAA content was recorded in 'Vetra' (VII organogenesis stage) after long -day 130+20 day vernalization and growth in greenhouse, when stem elongation started. At that time IAA content was 1.5 times bigger in 'Vètra' accumulated than that in 'Punia', which has only been achieved tillering stage (IV organogenesis stage). IAA induces growth and morphogenesis (Korasick et al., 2013).

IBA discovered quite recently and is more effective auxin than IAA (Mok and Mok, 2001). IAB content recorded significantly 3.2 times bigger in 'Vètra' than in 'Punia' after 130+20 day vernalisation and growth in greenhouse, when stem elongation occurred.

Though cytokines regulate some growth and development processes, involving cell division, apical domination, chloroplast biogenesis, photomorphogenetic development, shoot differentiation and anthocyanin synthesis (Mok and Mok, 2001; Davies, 2004), however it was found only in trace amounts.

JA content was revealed significantly the biggest after treatment with 130 long-days and low positive temperatures at the end of the flowering induction, when Festulolium achieved stem elongation (VII organogenesis stage). At this moment JA content was 1.4 times bigger in 'Vètra' than that in 'Punia'. However, JA content existed 1.6 times bigger after 90 long-days vernalisation in 'Punia' thant in 'Vètra'. JA is important for plant resistance to pathogens and different stressors (Cheong and Choi, 2003).

Saccharides present basic primary metabolites involved in growth and development. They are involved in numerous metabolic reactions performing their regulation (Halford et al., 2010). The data revealed that saccharides content significantly depended on photoperiod during vernalisation (Table 2-7).

'Vètra' and 'Punia' accumulated significantly the biggest sucrose content of 8.85 and $8.15 \mu \mathrm{g} \mathrm{g}^{-1}$, respectively, after 90 short-days vernalisation at tillering stage (Table 2, 5). Generative development started when plants after vernalisation were moved to greenhouse for 20 days. Consequently, saccharides content decreased. Regular seasonal changes (photoperiod, temperature) are potential factors that control the transition to flowering. Sucrose is the main saccharide which content increases in plant early development, however only temporarily. Leaves respond to specific effect of inducing photoperiod increasing sucrose content which is transmitted to apical meristems and induce them (Koch, 2004).

Table 2. Impact of 90 day vernalisation and different photoperiod on 'Vètra' saccharides content $\left(\mu \mathrm{g} \mathrm{g}^{-1}\right)(*-\mathrm{p}<0.05)$

\begin{tabular}{lcccccc}
\hline Saccharide & \multicolumn{3}{c}{90 day } & \multicolumn{2}{c}{$90+20$ day in greenhouse } \\
\cline { 2 - 7 } & $16 \mathrm{~h}-20^{\circ} \mathrm{C}$ & $8 \mathrm{~h}-4^{\circ} \mathrm{C}$ & $16 \mathrm{~h}-4^{\circ} \mathrm{C}$ & $16 \mathrm{~h}-20^{\circ} \mathrm{C}$ & $8 \mathrm{~h}-4^{\circ} \mathrm{C}$ & $16 \mathrm{~h}-4^{\circ} \mathrm{C}$ \\
\cline { 2 - 7 } & VII & IV & II & VII & V & V \\
& Stem elongation & Tillering & Leaf development & Stem elongation & Booting & Booting \\
\hline Sucrose & $5.85^{*}$ & $8.85^{*}$ & $7.04^{*}$ & 5.85 & $0.37^{*}$ & $0.21^{*}$ \\
Glucose & $6.44^{*}$ & 18.0 & $4.09^{*}$ & $6.44^{*}$ & $0.13^{*}$ & $0.14^{*}$ \\
Mannose & $1.43^{*}$ & 10.8 & $1.46^{*}$ & $1.43^{*}$ & $0.36^{*}$ & $0.26^{*}$ \\
Fructose & $1.77^{*}$ & $64.9^{*}$ & $4.08^{*}$ & $1.77^{*}$ & $1.21^{*}$ & $0.88^{*}$ \\
\hline
\end{tabular}

Sucrose accumulation significantly decreased in 'Vètra' after 110 long-day vernalisation due to intensive growth of stem (Table 3). Nonetheless, fructose rate was $64.9 \mu \mathrm{g} \mathrm{g}^{-1}$ after 110 short-day vernalisation and 12.7 times than that after 130 short-day vernalisation (Table 4). After further 20 days in greenhouse, plant generative development started and significantly increased sucrose, glucose and fructose content in 12.2, 3.8 and 2.5 times respectively than that after 110 long-day vernalisation (Table 3 ).

Table 3. Impact of 110 day vernalisation and different photoperiod on 'Vètra' saccharides content $\left(\mu \mathrm{g} \mathrm{g}^{-1}\right)(*$ - $\mathrm{p}<0.05)$

\begin{tabular}{lcccccc}
\hline Saccharide & \multicolumn{3}{c}{110 day } & \multicolumn{3}{c}{$110+20$ day in greenhouse } \\
\cline { 2 - 7 } & $16 \mathrm{~h}-20^{\circ} \mathrm{C}$ & $8 \mathrm{~h}-4^{\circ} \mathrm{C}$ & $16 \mathrm{~h}-4^{\circ} \mathrm{C}$ & $16 \mathrm{~h}-20^{\circ} \mathrm{C}$ & $8 \mathrm{~h}-4^{\circ} \mathrm{C}$ & $16 \mathrm{~h}-4^{\circ} \mathrm{C}$ \\
\cline { 2 - 7 } & $\mathrm{X}$ & III & III & $\mathrm{X}$ & $\mathrm{V}$ & $\mathrm{V}$ \\
& Milky stage & Tillering & Tillering & Milky stage & Stem elongation & Stem elongation \\
\hline Sucrose & 0.19 & 0.25 & $0.07^{*}$ & 0.19 & $0.23^{*}$ & $0.86^{*}$ \\
Glucose & 0.21 & 0.29 & 0.52 & $0.22^{*}$ & $1.11^{*}$ & 0.63 \\
Mannose & 0.13 & 0.11 & 0.17 & $0.13^{*}$ & 0.26 & 0.16 \\
Fructose & 0.50 & $0.40^{*}$ & $0.89^{*}$ & 0.49 & $1.03^{*}$ & $0.61^{*}$ \\
\hline
\end{tabular}

Total saccharides content decreased by 4.1 times, but increased glucose, manose and fructose in 'Vètra' after 130 short-days + 20 day in greenhouse due to initiation of generative development as compared with that of 130 short-day vernalisation (Table 4). However, 'Vetra' accumulated significantly higher rates of sucrose after 130+20 long-days $\left(0.31 \mu \mathrm{g} \mathrm{g}^{-1}\right)$ than that of $130+20$ short-days vernalisation $\left(0.16 \mu \mathrm{g} \mathrm{g}^{-1}\right)$. 
Table 4. Impact of 130 day vernalisation and different photoperiod on 'Vètra' saccharides content $\left(\mu \mathrm{g} \mathrm{g}^{-1}\right)(*$ - $\mathrm{p}<0.05)$

\begin{tabular}{lcccccc}
\hline Saccharide & \multicolumn{3}{c}{130 day } & \multicolumn{3}{c}{$130+20$ day in greenhouse } \\
\cline { 2 - 7 } & $16 \mathrm{~h}-20^{\circ} \mathrm{C}$ & $8 \mathrm{~h}-4^{\circ} \mathrm{C}$ & $16 \mathrm{~h}-4^{\circ} \mathrm{C}$ & $16 \mathrm{~h}-20^{\circ} \mathrm{C}$ & $8 \mathrm{~h}-4^{\circ} \mathrm{C}$ & $16 \mathrm{~h}-4^{\circ} \mathrm{C}$ \\
\cline { 2 - 7 } & $\begin{array}{c}\mathrm{XII} \\
\text { Hard dough }\end{array}$ & $\begin{array}{c}\text { III } \\
\text { Tillering }\end{array}$ & $\begin{array}{c}\text { III } \\
\text { Tillering }\end{array}$ & $\begin{array}{c}\text { IX } \\
\text { Flowering }\end{array}$ & $\begin{array}{c}\text { VIII } \\
\text { Heading }\end{array}$ & Stem elongation \\
\hline Sucrose & 0.58 & 0.66 & 0.18 & $0.29^{*}$ & $0.16^{*}$ & $0.31^{*}$ \\
Glucose & $1.00^{*}$ & 0.27 & $0.05^{*}$ & 0.17 & $1.00^{*}$ & 0.07 \\
Mannose & $0.18^{*}$ & 0.11 & $0.07^{*}$ & 0.03 & $0.21^{*}$ & 0.07 \\
Fructose & $1.05^{*}$ & 0.32 & $0.07^{*}$ & 0.14 & $1.18^{*}$ & $0.05^{*}$ \\
\hline
\end{tabular}

Significantly the maximal sucrose content of $8.15 \mu \mathrm{g} \mathrm{g}{ }^{-1}$ was documented in 'Punia' after 90 short-days vernalisation (Table 5). 90 short-days vernalisation induced significantly the biggest accumulation of glucose, mannose and fructose. Saccharides content significantly decreased after plants were moved for 20 days to greenhouse, because they started intensive growth from tillering (III organogenesis stage) to stem elongation (V organogenesis stage).

Table 5. Impact of 90 day vernalisation and different photoperiod on 'Punia' saccharides content $\left(\mu \mathrm{g} \mathrm{g}^{-1}\right)(*-\mathrm{p}<0.05)$

\begin{tabular}{lcccccc}
\hline Saccharide & \multicolumn{3}{c}{90 day } & \multicolumn{3}{c}{$90+20$ day in greenhouse } \\
\cline { 2 - 7 } & $16 \mathrm{~h}-20^{\circ} \mathrm{C}$ & $8 \mathrm{~h}-4^{\circ} \mathrm{C}$ & $16 \mathrm{~h}-4^{\circ} \mathrm{C}$ & $16 \mathrm{~h}-20^{\circ} \mathrm{C}$ & $8 \mathrm{~h}-4^{\circ} \mathrm{C}$ & $16 \mathrm{~h}-4^{\circ} \mathrm{C}$ \\
\cline { 2 - 7 } & VII & III & III & VII & V & V \\
& Stem elongation & Tillering & Tillering & Stem elongation & Booting & Booting \\
\hline Sucrose & $6.01^{*}$ & $8.15^{*}$ & $7.09^{*}$ & 6.01 & 0.79 & 0.34 \\
Glucose & $6.73^{*}$ & 5.27 & $15.51^{*}$ & $6.73^{*}$ & 0.17 & 0.27 \\
Mannose & $3.51^{*}$ & 0.73 & $10.6^{*}$ & 3.51 & 0.16 & 0.33 \\
Fructose & $2.18^{*}$ & $6.88^{*}$ & $38.9^{*}$ & $2.18^{*}$ & 0.39 & 0.49 \\
\hline
\end{tabular}

After 110 days vernalisation 'Punia'achieved tillering stage (Table 6). When it was moved to greenhouse for 20 days, plants started intensive grow and rapidly dissimilated saccharides especially at long-day photoperiod. Therefore saccharides content decreased, mainly glucose and fructose content in 52 and 31.1 times than that of 110 vernalisation. Sucrose decreased by 5.8 times after 20 days in greenhouse.

Table 6. Impact of 110 day vernalisation and different photoperiod on 'Punia' saccharides content $\left(\mu \mathrm{g} \mathrm{g}^{-1}\right)(*-\mathrm{p}<0.05)$

\begin{tabular}{lcccccc}
\hline Saccharide & \multicolumn{3}{c}{110 day } & \multicolumn{3}{c}{$110+20$ day in greenhouse } \\
\cline { 2 - 7 } & $16 \mathrm{~h}-20^{\circ} \mathrm{C}$ & $8 \mathrm{~h}-4^{\circ} \mathrm{C}$ & $16-4^{\circ} \mathrm{C}$ & $16 \mathrm{~h}-20^{\circ} \mathrm{C}$ & $8 \mathrm{~h}-4^{\circ} \mathrm{C}$ & \multicolumn{1}{c}{$16 \mathrm{~h}-4^{\circ} \mathrm{C}$} \\
\cline { 2 - 7 } & $\mathrm{X}$ & $\mathrm{III}$ & $\mathrm{III}$ & $\mathrm{X}$ & $\mathrm{V}$ & $\mathrm{V}$ \\
\cline { 2 - 7 } & Milky stage & Tillering & Tillering & Milky stage & Booting & Booting \\
\hline Sucrose & $0.33^{*}$ & $0.58^{*}$ & $0.35^{*}$ & $0.33^{*}$ & $0.13^{*}$ & $0.06^{*}$ \\
Glucose & 4.40 & $3.48^{*}$ & $2.60^{*}$ & $4.41^{*}$ & $0.26^{*}$ & $0.05^{*}$ \\
Mannose & 1.88 & $0.52^{*}$ & $0.58^{*}$ & $1.88^{*}$ & $0.12^{*}$ & $0.06^{*}$ \\
Fructose & $7.02^{*}$ & $4.39^{*}$ & $3.73^{*}$ & $7.02^{*}$ & $0.52^{*}$ & $0.12^{*}$ \\
\hline
\end{tabular}

Saccharides content was lower in 'Punia' after 130 long-day vernalisation that after short-day (Table 7). This decrease might be achieved due to start of development of inflorescence components (IV organogenesis stage) after 130 long-day vernalisation. Additional decrease of saccharides content was observed while further keeping plants in 20 days in greenhouse though growth stage did not changed. These compounds possibly were used for the formation of new shoots. Saccharides content was also lower in 'Punia' than in 'Vetra'.

Table 7. Impact of 130 day vernalisation and different photoperiod on 'Punia' saccharides content $\left(\mu \mathrm{g} \mathrm{g}^{-1}\right)\left(*_{-} \mathrm{p}<0.05\right)$

\begin{tabular}{lcccccc}
\hline Saccharide & \multicolumn{3}{c}{130 day } & \multicolumn{3}{c}{$130+20$ day in greenhouse } \\
\cline { 2 - 7 } & $16 \mathrm{~h}-20^{\circ} \mathrm{C}$ & $8 \mathrm{~h}-4^{\circ} \mathrm{C}$ & $16 \mathrm{~h}-4^{\circ} \mathrm{C}$ & $16 \mathrm{~h}-20^{\circ} \mathrm{C}$ & $8 \mathrm{~h}-4^{\circ} \mathrm{C}$ & $16 \mathrm{~h}-4^{\circ} \mathrm{C}$ \\
\cline { 2 - 7 } & XII & III & IV & V & III & IV \\
\cline { 2 - 7 } & Hard dough & Tillering & Tillering & Booting & Tillering & Tillering \\
\hline Sucrose & 0.13 & 0.44 & 0.06 & $0.35^{*}$ & $0.17^{*}$ & $0.11^{*}$ \\
Glucose & 0.16 & 1.57 & 0.38 & $0.27^{*}$ & $1.10^{*}$ & $0.04^{*}$ \\
Mannose & 0.09 & 0.24 & 0.09 & 0.13 & $0.25^{*}$ & $0.04^{*}$ \\
Fructose & 0.36 & 2.47 & 0.37 & $0.69^{*}$ & $1.22^{*}$ & $0.04^{*}$ \\
\hline
\end{tabular}

\section{CONCLUSIONS}

The data revealed that different duration and conditions of vernalisation impacted metabolites content of Festulolium cultivars. Low temperatures and short-day photoperiod during different duration of vernalisation conditioned significantly the maximal total phytohormones content. Moreover, composition of accumulated phytohormones varied in dependence with plant growth and organogenesis stages, which responded to vernalisation conditions. Between them only traces of cytokines were detected in leaves of the both Festulolium cultivars. However the maximal gibberellins content was recorded after 90 long-day vernalisation. Gibberellins stimulate leaf growth, therefore their bigger amounts were 
detected in Festulolium cultivars at tillering stage (IV organogenesis stage). Phytohormones content decreased after the longest vernalisation period of 130 days when plants has achieved stem elongation and further growth stages.

Content of primary metabolites, i.e. saccharides, was different in assessed Festulolium cultivars and depended on their vernalisation conditions. Low temperatures stimulated accumulation of monosaccharides in 'Punia'in tilleringbooting stages. However, 110 long-days vernalisation stimulated accumulation of saccharides content in 'Vètra'. Summarising, low temperature stimulate saccharides accumulation at preceding growth stages.

\section{REFERENCES}

1. Bernier, G., Havelange, A., Housa, C., Petitjean, A., Lejeune, P. 1993. Physiological signals that induce flowering. The Plant Cell. Vol. 5, Iss. 10, pp. 1147-1155. https://doi.org/10.1105/tpc.5.10.1147

2. Cheng, W.H., Endo, A., Zhou, L., Penney, J., Chen, H.C.,Arroyo, A., Leon, P., Nambara, E., Asami, T., Seo, M., Koshiba, T., Sheen, J. 2002. A unique short-chain dehydrogenase/reductase in Arabidopsis glucose signaling and abscisic acid biosynthesis and functions. The Plant Cell. Vol. 14, Iss. 11, pp. 2723-2743. https://doi.org/10.1105/tpc.006494

3. Cheong, J., Choi, Y.D. 2003. Methyl jasmonate as a vital substance in plants. Trends in Genetics. Vol. 19, Iss. 7, pp. $409-413$. https://doi.org/10.1016/S0168-9525(03)00138-0

4. Davies, P.J. 2004. Plant hormones: biosynthesis, signal transduction, action. Kluwer Academic Press, the Netherlands

5. Duchovskis, P. 2004. Flowering initiation of wintering plants. Sodininkystè ir daržininkystè. Vol. 23, No. 2, pp. 3-11.

6. Gibson, S. I. 2004. Sugar and phytohormone response pathways: navigating a signalling network. Journal of Experimental Botany. Vol. 55, Iss. 395, pp. 253-264. https://doi.org/10.1093/jxb/erh048

7. Halford, N.G., Curtis, T.Y., Muttucumaru, N., Postles, J., Mottram, D.S. 2011. Sugars in crop plants. Annals of Applied Biology. Vol. 158, Iss. 1, pp. 1-25. https://doi.org/10.1111/j.1744-7348.2010.00443.x

8. Javid, M.G., Sorooshzadeh, A., Moradi, F., Sanavy, S.A.M.M., Allahdadi, I. 2011. The role of phytohormones in alleviating salt stress in crop plants. Australian Journal of Crop Science. Vol. 5, Iss. 6, pp. 58-67.

9. Koch, K. E. 2004. Sucrose metabolism: regulatory mechanisms and pivotal roles in sugar sensing and plant development. Current Opinion in Plant Biology. Vol. 7, Iss. 3, pp. 235-246. https://doi.org/10.1016/j.pbi.2004.03.014

10. Korasick, D.A., Enders, T.A., Strader, L.C. 2013. Auxin biosynthesis and storage forms. Journal of Experimental Botany. Vol. 64 , Iss. 9, pp. 2541-2555. https://doi.org/10.1093/jxb/ert080

11. Koussevitzky, S., Nott, A., Mockler, T.C., Hong, F., Sachetto-Martins, G., Surpin, M., Chory, J. 2007. Signals from chloroplasts converge to regulate nuclear gene expression. Science. Vol. 316, Iss. 5825, pp. 715-719. https://doi.org/10.1126/science

12. Kuperman, F.M., Rzhanova, E.I., Murashew, V.V., Lvova, I.N., Sedova, E.A., Ahundova, V.A., Scherbina, I.P. Development biology of crops. Vysshaya shkola, Moscow, 1982, 343 p. [In Russian]

13. Magome, H., Yamaguchi, S., Hanada, A., Kamiya, Y., Odadoi, K. 2004. Dwarf and delayed-flowering 1, a novel Arabidopsis mutant deficient in gibberellins biosynthesis because of over expression of a putative AP2 transcription factor. The Plant Journal. Vol. 37, Iss. 5, pp. 720-729. https://doi.org/10.1046/j.1365-3040.2000.00643.x

14. Michaels, S.D., Amasino, R.M. 2000. Memories of winter: vernalization and the competence to flower. Plant, Cell and Environment. Vol. 23, Iss.. 11, pp.1145-1153. https://doi.org/10.1046/j.1365-3040.2000.00643.x

15. Mok, Dw., Mok, M.C. 2001. Cytokinin metabolism and action. Annual Review of Plant Physiology and Plant Molecular Biology. Vol.52, pp. 89-118. https://doi.org/10.1146/annurev.arplant.52.1.89

16. Nacionalinis augalų veislių sąrašas. VAT, ŽŪM, 2008. [In Lithuanian]

17. Samuolienè, G., Šabajevienė, G., Urbonavičiūte, A., Duvhovskis, P. 2008. Plant morphogenesis and flowering initiation. Sodininkyste ir daržininkystè. Vol. 27, No. 3, pp. 241-251. [In Lithuanian] 